\title{
Piezoelectric energy conversion in windmills
}

\section{Author: V. Hugo Schmidt}

This is a postprint of an article that originally appeared in Ultrasonics Symposium Proceedings on October 20-23, 1992. http://dx.doi.org/10.1109/ULTSYM.1992.275842

Schmidt, V.H. "Piezoelectric Energy Conversion in Windmills." IEEE 1992 Ultrasonics Symposium Proceedings (October 20-23, 1992). doi: 10.1109/ultsym.1992.275842.

Made available through Montana State University's $\underline{\text { ScholarWorks }}$ 


\title{
PIEZOELECTRIC ENERGY CONVERSION IN WINDMILLS*
}

\author{
V. Hugo Schmidt
}

Physics Department, Montana State University, Bozeman, MT 59717

\begin{abstract}
This paper reviews theory and practice for wind generators based on piezoelectric polymers. The basic piezoelectric equations are applied to bimorphs set into mechanical oscillation by the wind. The importance of incorporating mechanical and electrical resonance into the design is emphasized. The design and performance of two rotating and one oscillating generators are described, and performance is compared with theory. Design improvements are proposed and future prospects are evaluated.
\end{abstract}

\section{INTRODUCTION}

\section{Wind Energy Overview}

Generation of electrical energy from windpower has a long history. Numerous small wind generators were used on farms in the early and middle parts of this century, until rural electrification almost eliminated them. When the energy crisis hit in the early 1970 's, government programs focused mainly on research on very large machines and on tax incentives for alternate energy production which encouraged medium-sized machines, especially in California. The large machines in several cases had structural failures. The California wind farms were criticized as being tax farms, but now with improved and massproduced machines they are considered by some utilities as part of their electrical generation mix. Wind electrical generation is growing steadily and has a promising future (1), because machines are becoming more reliable, and more efficient thanks to sophisticated aerodynamic analysis.
Reasons for considering piezoelectric wind generators

With conventional wind generators showing such improvements and promise, why should piezoelectric wind generators be considered? A major drawback to conventional generators is the large high-speed rotor, which poses a serious safety problem except where people can be excluded. Even in such locations, there is concern that eagles and other birds are killed by flying into the rotors. Whereas rotating electromagnetic motors and generators are efficient and compact, piezoelectric devices excel in oscillating applications. In the following discussion we keep in view the possibility of a large piezoelectric wind generator, oscillating at high frequency with small amplitude and thus not posing serious safety problems.

\section{Outline of this paper}

We begin with the theory (2) for piezoelectric power generation, giving the basic equations and applying them to bimorphs. Then we discuss the advantages of employing mechanical and electrical resonance in the system design. We present typical properties of piezoelectric polymers and predict system performance based on these properties.

We next describe the design and performance of the two rotating and one oscillating piezoelectric polymer wind generators that we have constructed, and compare performance with theoretical predictions. Finally we explain proposed improved designs and discuss the future prospects for this type of generator. 


\section{THEORY}

\section{Basic Piezoelectric Equations}

To calculate the power from a given generator design for a given blade deflection amplitude and oscillation frequency, we begin with the piezoelectric equations

$$
\begin{aligned}
& \delta_{11}=S_{11} / Y_{111}+d_{31} E_{3}, \\
& D_{3}=K_{0} K_{33} E_{3}+d_{31} S_{11} .
\end{aligned}
$$

For nonpiezoelectric materials for which $d_{31}=0$, these are just uncoupled mechanical and electrical equations. We have omitted pyroelectric and thermal expansion terms which are not very important. They must be considered in an exact analysis because our operating frequencies are high enough that adiabatic rather than isothermal conditions obtain. The subscripts 1 and 3 refer to the directions described above. From here on, these subscripts will be omitted. We eliminate stress $S$ from Eqs. (1) and (2) in favor of the more easily measured strain $\delta$, and solve them simultaneously to obtain

$$
\mathrm{D}=\mathrm{K}_{0} \mathrm{~K}\left(1-\mathrm{Yd}^{2} / \mathrm{K}_{0} \mathrm{~K}\right) \mathrm{E}+\mathrm{Yd} \delta \approx \mathrm{K}_{0} \mathrm{KE}+\mathrm{Yd} \delta .
$$

Here $\mathrm{Yd}^{2} / \mathrm{K}_{0} \mathrm{~K}$ is the dimensionless electromechanical coupling constant $k^{2}$, which is small $(=0.0088)$ for $\mathrm{PVF}_{2}$ and can be neglected in this context.

In a piezoelectric generator operating in a bending mode, each volume element obeys the above equations, and the entire generator is equivalent to a slab of piezoelectric material as represented in Fig. 1, with electroded surfaces of area $w b$, one grounded and the other connected to terminal $T$. If the generator is driven with constant mechanical amplitude and frequency, the slab can be replaced by the equivalent generator consisting of an ideal emf $\varepsilon$ in series with a capacitor $C$ and resistor $R_{e}$ as derived below and shown in Fig. 1.

We assume a sinusoidal strain $\delta=\delta_{m} e^{j \omega t}$. The emf $\varepsilon$ then is the open-circuit voltage found from Eq. (3) by setting $D=0$ :

$$
\varepsilon=h E=-\left(\mathrm{Yhd} / \mathrm{K}_{0} \mathrm{~K}\right) \delta \text {, }
$$
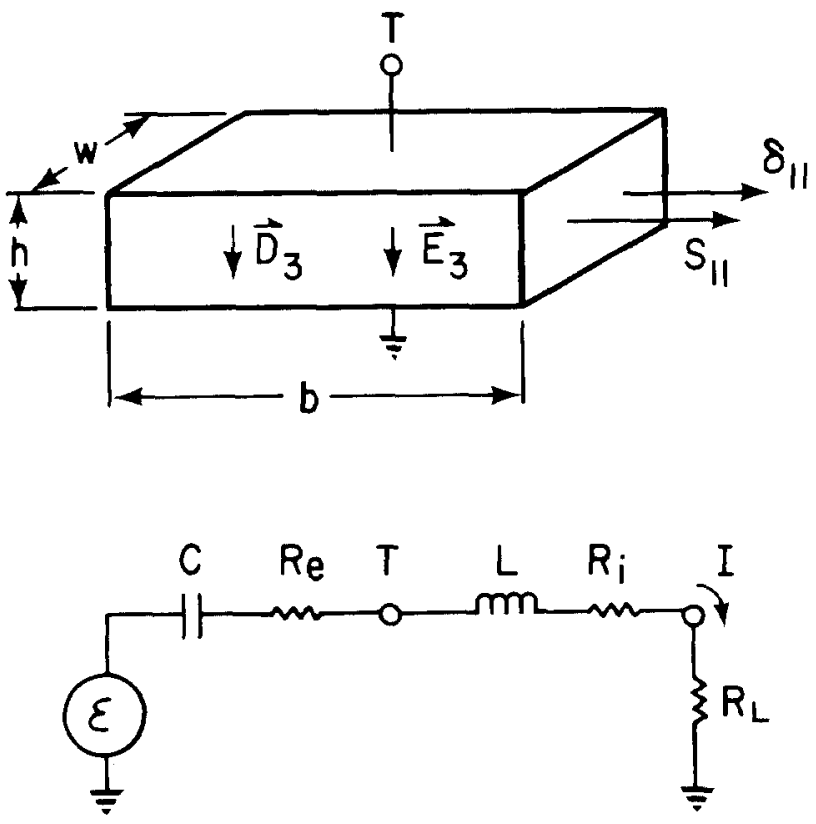

Figure 1. Slab of electroded piezoelectric polymer representing piezoelectric generator operating in $d_{31}$ mode, and its equivalent circuit coupled at terminal $T$ to a resonant inductor and a load $R_{L}$. Symbols are explained in text.

where $h$ is the thickness of the sheet.

The current is given by $\mathrm{I}=-\mathrm{wbdD} / \mathrm{dt}$, so the short-circuit current $I_{s}$ found by setting $E=0$ in $E q$. (3) is

$$
I_{s}=-j \omega w b Y d \delta=\varepsilon / Z_{s} .
$$

We see that while for a given maximum strain $\delta_{m}$ the emf is independent of the angular frequency $\omega$, the current is proportional to $\omega$, demonstrating the advantage of designing devices that oscillate at high frequency.

Finally, from Eqs. (4) and (5), the source impedance is

$$
Z_{\mathrm{s}}=\varepsilon / T_{\mathrm{s}}=-\mathrm{jh} / \omega \mathrm{K}_{0} \mathrm{Kwb}=-\mathrm{j} / \omega \mathrm{C} \text {, }
$$

so the source impedance is simply the capacitive reactance of the slab's capacitance $C$, where the small lossy part of the dielectric permittivity has been neglected. This is justified if the generator is connected directly to a resistive load. The lossy 
component must be considered if the capacitive component of the source impedance is resonated away by a series inductor to increase the output as discussed below.

If the generator (or group of generators forced mechanically to oscillate in phase) is connected to an inductor of the correct value to give series resonance at the operating frequency, the new source impedance will just be the combined resistance $R_{s}=R_{e}+R_{i}$ of the generator resistance $R_{e}$ and inductor resistance $R_{i}$ shown in Fig. 1 . From the value 50 chosen above for $Q_{e}=1 / \omega C_{e}$ for $\mathrm{PVF}_{2}$ and choosing a value of 33 for $\mathrm{Q}_{i}=\omega L / R_{i}$ of the series inductor at $1 \mathrm{kHz}$, the combined source $Q$ value is $Q_{s}=20$. Thus $R_{s}$ is $1 / 20$ of the magnitude of the capacitive source reactance $X_{c}=-j / \omega C$, where the capacitance $C=K_{0} K^{\prime} w b / h$ in accord with Eq. (6). Accordingly,

$$
\mathrm{R}_{\mathrm{s}}=\mathrm{h} / \mathrm{wbQ} \mathrm{K}_{\mathrm{s}} \mathrm{K}^{\prime} \omega
$$

is the source resistance of the generator when electrical resonance is employed.

We emphasize here that $L$ is an actual inductor, and not an equivalent inductor employed to analyze piezoelectric resonance. The electrical resonance described above has approximate angular frequency $\omega=(\mathrm{LC})^{-1 / 2}$. Good designs should incorporate mechanical resonance at the same frequency to maximize amplitude of oscillation, but we assume here that this frequency is far from the piezoelectric resonance frequency of the material.

With the decreased source impedance given in Eq. (7), much larger currents are possible for the same emf and consequently the output power will increase accordingly. We must check to see whether this large current I can cause the breakdown field $E_{b}$ to be exceeded. The field is the voltage which is approximately $\mathrm{X}_{\mathrm{c}} \mathrm{I}$ for large $Q_{s}$, divided by the thickness $h$. The current $I$ is the emf $\varepsilon$ from Eq. (4) divided by $R_{s}+R_{L}=R_{s}(1+r)$, where $R_{L}$ is the load resistance and $r$ is the ratio of load to source resistance. Since the magnitude of the ratio $X_{c} / R_{s}=Q_{s}$, we have, using Eq. (4):

$$
E=Q_{s} \varepsilon / h(1+r)=Q_{s} Y d \delta / K_{0} K^{\prime}(1+r) .
$$

If $\delta$ is set at the yield strain $0.03, \mathrm{r}$ at 0 (short circuited output), and other parameters given above are substituted into Eq. (8), we obtain
$\mathrm{E}_{\max }=21.2 \times 10^{7} \mathrm{~V} / \mathrm{m}$. This is larger than the breakdown field of $E_{b}=3 \times 10^{7} \mathrm{~V} / \mathrm{m}$, so breakdown must be considered as a possible limitation on output power, as described below. If electrical resonance is not employed, then maximum field occurs for open-circuited load, in which case Eq. (4) applies. The field magnitude corresponding to the emf at yield strain found from Eq. (4) is defined as

$$
\mathrm{E}_{\mathrm{y}}=\varepsilon_{\mathrm{y}} / \mathrm{h}=\mathrm{Yd} \delta_{\mathrm{y}} / \mathrm{K}_{0} \mathrm{~K}^{\prime}=1.06 \times 10^{7} \mathrm{~V} / \mathrm{m},
$$

so breakdown will not limit the power output if electrical resonance is not employed.

\section{Theoretical Electrical Power Output}

For the electrically resonant case, the power $\mathrm{W}$ to the load per unit volume of $\mathrm{PVF}_{2}$ is limited either by mechanical yield or electrical breakdown, depending on polymer parameters and the inductor and load resistances. For the yield-limited case,

$$
\begin{gathered}
\mathrm{W}_{\mathrm{y}}=\mathrm{I}_{\mathrm{y}}{ }^{2} \mathrm{R}_{\mathrm{L}} / 2 \mathrm{wbh}=\left[\mathrm{E}_{\mathrm{y}} \mathrm{h} / \mathrm{R}_{\mathrm{s}}(1+\mathrm{r})\right]^{2} \mathrm{R}_{\mathrm{s}} \mathrm{r} / 2 \mathrm{wbh}(10) \\
=\mathrm{Q}_{\mathrm{s}} \mathrm{rK}_{0} \mathrm{~K} \cdot \omega \mathrm{E}_{\mathrm{y}}{ }^{2} / 2(1+\mathrm{r})^{2}
\end{gathered}
$$

using Eq. (7) for $R_{s}$. Note that for increasing load ratio $r, W_{y}$ first increases linearly, peaks at $r=1$, and then decreases.

For the breakdown-limited case,

$$
\begin{gathered}
W_{b}=I_{b}{ }^{2} R_{L} / 2 w b h=\left(E_{b} / X_{C}\right)^{2} R_{s} r / 2 w b h \\
=\left(E_{b} / Q_{s} R_{s}\right)^{2} R_{s} r / 2 w b h=r K_{0} K^{\prime} w E_{b}^{2} / 2 Q_{s} .
\end{gathered}
$$

Note that $W_{b}$ increases linearly with $r$ for all $r$. If there is a crossover from breakdown-limited to yield-limited power, it must occur at the $r$ value $r_{x}$ at which $W_{b}=W_{y}$. From Eqs. (10) and (11), this occurs at

$$
r_{x}=Q_{s} E_{y} / E_{b}-1 .
$$

There are three possibilities for the loaddependence of the power output per unit volume.

First, if Eq. (12) indicates negative $r_{x}$, the yieldlimited case of Eq. (10) is valid for all $r$, and the maximum power at $r=1$ is 


$$
\mathrm{W}_{\mathrm{my}}=\mathrm{Q}_{\mathrm{s}} \mathrm{K}_{0} \mathrm{~K}^{\prime} \omega \mathrm{E}_{\mathrm{y}}^{2} / 8 \text {. }
$$

Second, if $0<r_{x}<1$, the maximum power is governed by Eq. (11) for $r<r_{x}$ and Eq. (10) for $r>r_{x}$, and the maximum power still occurs at $r=1$ and is still given by Eq. (13).

Third, if $r_{x}>1$, the maximum power is still governed by Eq. (11) or (10) as described above, but the maximum power occurs at $r=r_{x}$ and is given by

$$
W_{m x}=\left(K_{0} K^{\prime} \omega E_{b} / 2\right)\left(E_{y}-E_{b} / Q_{s}\right) .
$$

For the parameters given above, we are in the third case, with $r_{x}=6.06$. For a frequency of $1 \mathrm{kHz}$, somewhat below the frequency at which $\mathrm{K}^{n}$ starts increasing rapidly for $\mathrm{PVF}_{2}, \mathrm{~W}_{\mathrm{mx}}=91 \mathrm{~W} / \mathrm{cm}^{3}$. This is a significant power level, and so possible overheating of the material must be considered in the design process, even after the operating power level is reduced to provide a factor of safety against yield and breakdown. The power curve as a function of load resistance is shown in Fig. 2.

If electrical resonance is not employed, the power is limited by yield and Eq. (13) applies for

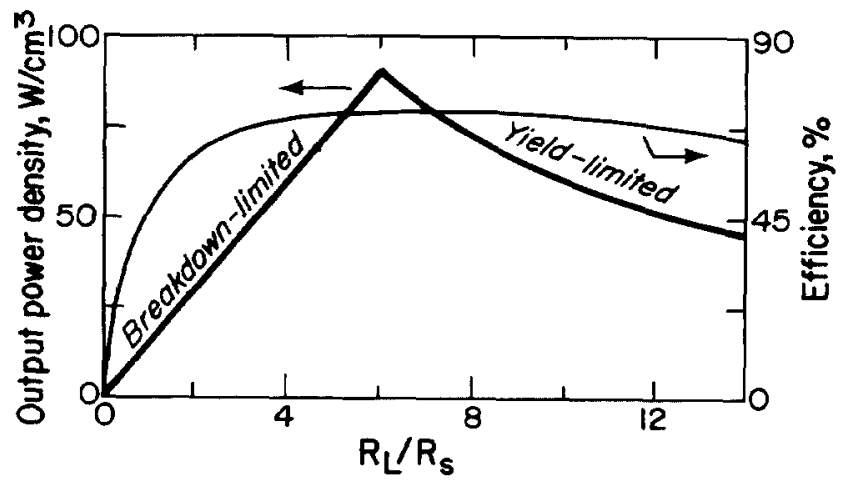

Figure 2. Theoretical maximum electrical power output density and efficiency for a $\mathrm{PVF}_{2}$-based generator osclllated mechanically at $1 \mathrm{kHz}$. maximum power, with $Q_{s}$ replaced by 2 . For maximum power, the load resistance is numerically equal to the capacitive source impedance. The 2 occurs because the load is $90^{\circ}$ out of phase with the source impedance. Thus the voltage across each element is $\varepsilon / 2^{1 / 2}$ instead of $\varepsilon / 2$, and the square of voltage which is proportional to power is twice as great. The maximum power for the above parameters is $19 \mathrm{~W} / \mathrm{cm}^{3}$. Thus adding a series inductor to provide electrical resonance increases the power per unit volume of $\mathrm{PVF}_{2}$ almost fivefold.

\section{Efficiency}

The efficiency is the power to the load, divided by the sum of the load power and the electrical and mechanical power losses, which must add up to the mechanical input power. In each case we consider power per unit volume. The three power and loss components have the same ratios independent of amplitude of oscillation, or of whether yield or breakdown limits power output. Accordingly, we can drop the subscript y from Eq. (10) and use Eq. (8) to obtain the output power $W_{0}$ in terms of the strain $\delta$ :

$$
W_{o}=Q_{s} r \omega Y^{2} d^{2} \delta^{2} / 2 K_{0} K \cdot(1+r)^{2}
$$

The electrical loss power $W_{\mathrm{el}}$ is given by

$$
\mathrm{W}_{\mathrm{el}}=\mathrm{W}_{\mathrm{o}} \mathrm{R}_{\mathrm{s}} / \mathrm{R}_{\mathrm{L}}=\mathrm{W}_{\mathrm{o}} / \mathrm{r}
$$

The mechanical loss power $\mathrm{W}_{\mathrm{ml}}$ is the product of the angular frequency $\omega$ and the mean energy loss per radian, which in turn is the energy stored divided by the mechanical quality factor $Q_{m}$. The energy stored per unit volume, in a formula analogous to that for the energy stored in a spring, is $\mathrm{Y} \delta^{2} / 2$. Thus, $\mathrm{W}_{\mathrm{ml}}$ becomes

$$
\mathrm{W}_{\mathrm{ml}}=\omega \mathrm{Y} \delta^{2} / 2 \mathrm{Q}_{\mathrm{m}}
$$

The efficiency $q$ then can be written as

$$
\begin{aligned}
\mathrm{r}=[1 & {\left[\mathrm{W}_{\mathrm{el}} / \mathrm{W}_{\mathrm{o}}+\mathrm{W}_{\mathrm{ml}} / \mathrm{W}_{\mathrm{o}}\right]^{-1} } \\
& =\left[1+\mathrm{r}^{-1}+(\mathrm{r}+1)^{2} / \mathrm{rF}\right]^{-1}
\end{aligned}
$$

in which $\mathrm{F}$ is a "Figure of merit" for the generator, given by 


$$
F=Q_{m} Q_{s} k^{2}
$$

and $\mathrm{k}^{2}$ is the electromechanical coupling constant $\mathrm{Yd}^{2} / \mathrm{K}_{0} \mathrm{~K}$ which is 0.0088 for $\mathrm{PVF}_{2}$. For our chosen values of 200 for $Q_{m}$ and 20 for $Q_{s}$, the figure of merit is 35.2 , much larger than $\mathrm{k}^{2}$ itself. At the load resistance ratio $r_{x}=6.06$ giving maximum power, the efficiency is 0.715 , or $71.5 \%$. As seen in Fig. 2, the efficiency is quite flat over a large range of $r$. It is maximum at $r_{m}=(F+1)^{g / h}=6.02$, which is coincidentally very near $r_{x}$.

If no series inductor is used to achieve resonance, the value of $Q_{s}=2$ discussed above must be used in Eq. (18). At maximum power of 19 $W / \mathrm{cm}^{3}, r=Q_{e}=50$, and efficiency $\mathrm{q}=0.0633$, or only $6.33 \%$. The efficiency peaks at $36.0 \%$ for $r_{m}=2.13$, but here the power density is very low, only 1.6 $\mathrm{W} / \mathrm{cm}^{3}$.

If the generator system is synchronized to the line and its output is fed into the $60 \mathrm{~Hz}$ line instead of a load resistor $R_{L}$, the above power output and efficiency equations still hold so long as an inductor is used to achieve electrical resonance. One simply replaces $R_{L}$ with $V / I$, where $V$ is line voltage and $I$ is current. At $60 \mathrm{~Hz}$ an inductor $Q_{i}$ of only 20 can be expected, but $Q_{e}$ remains at 50 , so the combined $Q_{s}$ becomes 14.3 and $r_{x}$ in Eq. (12) becomes 4.05. Then the maximum power density from $\mathrm{Eq}$. (14) becomes $5.1 \mathrm{~W} / \mathrm{cm}^{3}$ and the efficiency from Eq. (18) becomes $67 \%$. At this $r_{x}$ the ratio $\varepsilon / V=\left(R_{s}-R_{L}\right) / R_{L}=1+1 / r_{x}=1.25$, so maximum power is achieved with a generator emf $25 \%$ higher than line voltage.

At frequencies near $1 \mathrm{kHz}$, electrical power outputs approaching 100 watts per cubic centimeter of $\mathrm{PVF}_{2}$ at efficiencies near $70 \%$ can theoretically be obtained from mechanically-driven devices if the capacitive source impedance is resonated away by a series inductor. The load must be properly matched to the generator. The power output will be reduced by the product of the safety factors by which the device is operated below both the yield strain and electrical breakdown limits. Power and efficiency are tabulated below for different frequencies, with and without employing electrical resonance.

\begin{tabular}{cccc} 
Freq., $\mathrm{Hz}$ & Resonant? & Power, $\mathrm{W} / \mathrm{cm}^{3}$ & Efficiency,\% \\
1000 & yes & 91 & 72 \\
1000 & no & 19 & 6.3 \\
60 & yes & 5.1 & 67 \\
\hline
\end{tabular}

Table I. Maximum power density and corresponding efficiency for various operating conditions described more fully in text.

\section{GENERATOR DESIGNS AND PERFORMANCE}

\section{$\underline{\text { Savonius Rotor }}$}

The first "Savonius" design (3) we constructed and tested was of the Savonius type which has a vertical-axis rotor with an S-shaped cross section. Its construction and operation are schematized in Fig. 3. The standard Savonius rotor is of rigid construction, and our design would still rotate if the $\mathrm{PVF}_{2}$ bimorph were rigid, by virtue of the asymmetry introduced by the C-shaped aluminum end caps. However, the piezoelectric generation depends on flexure of the bimorph, which is curved one way in the illustration and will curve the other way (in the rotor reference frame) when the rotor has advanced one-half turn. The rotor will thus produce an ac voltage of frequency equal to the rotation frequency. The current flows from the metallized surfaces of the bimorph to the respective metal backstop tubes. Each tube is connected electrically to one bearing, so the power is brought out through the bearings, which act as slip rings. The backstop tube radius is sufficiently large so that even if a strong wind forces the bimorph to instantaneously adopt the curvature of one of the tubes, the $\mathrm{PVF}_{2}$ outer layers will not stretch or compress beyond the elastic limit.

This rotor operated satisfactorily in tests in a small wind tunnel capable of wind speeds to 25 $\mathrm{m} / \mathrm{s}(50 \mathrm{mph})$. Its electrical output voltage was as large as expected for the amplitude of bimorph oscillation which was observed stroboscopically. The output current corresponded to this voltage 


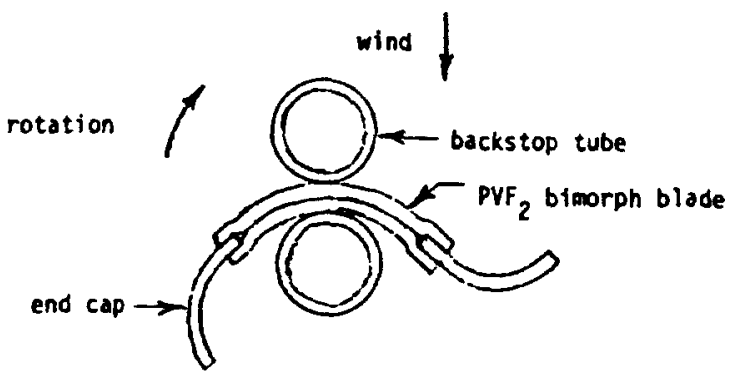

Fig. 3. Savonius rotor

divided by the capacitive source impedance of the bimorph. The largest amplitude oscillation could be expected if the rotation frequency equals the resonant oscillation frequency of the bimorph-cap arrangement as modified by centrifugal force effects in the rotating frame. We made no systematic studies of such resonance effects, other than to note that the oscillation amplitude was quite large at higher wind speeds, approximating the amplitude shown in the Figure.

\section{Lateral Blade Rotor}

The second "lateral blade" design (4) which is illustrated in Fig. 4 was intended to overcome two disadvantages of the Savonius design. One disadvantage is the small amount of active material compared to the rotor projected area seen by the wind stream. The other disadvantage is the lack of overspeed protection. This rotor is also designed for vertical axis operation. It has two cantilevermounted bimorph sheets tied together by a thin fishing line so that at zero rpm in no wind the bimorphs will bend to a cylindrical shape as shown, forming part of the cylindrical periphery of the rotor. The wind bends the upwind blade inward, allowing the downwind blade to partially straighten. This supplies the shape asymmetry needed to start rotation. At a certain rotational speed, given that one blade is instantaneously bent inward and the other outward from the equilibrium, the centrifugal force in the rotating frame which tries to accentuate the asymmetry is just as strong as the elastic force which tries to eliminate the asymmetry. This is the resonant rotational speed, at which the wind-induced oscillations reach large amplitude and cause a braking action which prevents overspeed. This speed-limiting effect was clearly seen in wind tunnel tests.

The rotor with piezoelectric polymer blades did not perform as well mechanically as when single sheets of electrically inert polymer were used for blades, and the electrical output was much less than predicted theoretically. The main problem was the unevenness of the glue thickness, which caused most of the bending to occur in small regions, rather than uniform bending as expected. The blades were made of two 110 micron Pennwalt uniaxial $\mathrm{PVF}_{2}$ sheets with two sheets of piezoelectrically inert viewgraph plastic inserted between them. The four sheets were glued together with "Superglue." The overall blade thickness was 500 microns, with $90 \mathrm{~mm}$ width at the base (attachment line) and tapered according to a cosine function to a length of $70 \mathrm{~mm}$. The radius from shaft centerline to the blades was $\mathbf{4 5}$ mm.

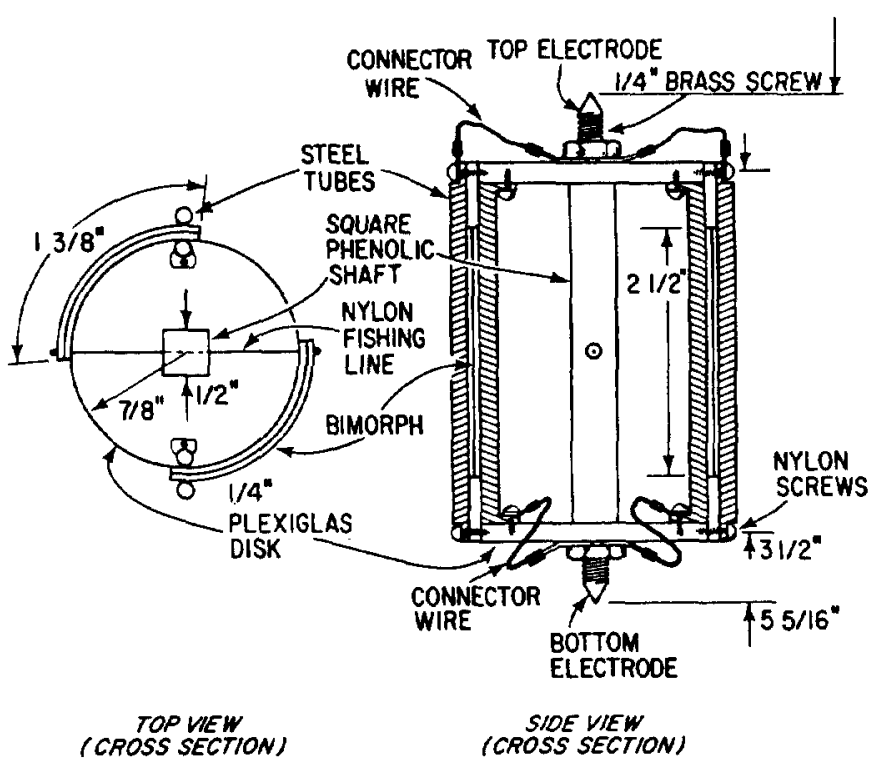

Fig. 4. Lateral blade rotor 
The best tip speed ratio (rotor linear speed at maximum radius compared to wind speed) was 0.50 at $11.6 \mathrm{~m} / \mathrm{s}$ wind speed and $1050 \mathrm{rpm}$ rotor speed. At $1350 \mathrm{rpm}$, corresponding to $15.9 \mathrm{~m} / \mathrm{s}$ wind speed, the maximum voltage attained was only 5 volts for a $0.43 \mathrm{megohm}$ load. The maximum power was 2.4 microwatts for a 400 kilohm load. This power is low for the amount of $\mathrm{PVF}_{2}$ used because, in addition to the problem of uneven glue thickness, the blade outer fibers at maximum deflection were strained only $1 / 6$ of the 0.03 yield strain, correponding to $1 / 36$ of the maximum possible power. A thicker blade with the same deflection would have come closer to the yield strain, and would also have more power output because of its greater volume.

\section{Oscillating Blade Generator}

Because of the complexity of rotating machines, especially given the requirement of small size in order to achieve high oscillation frequency, it seemed worthwhile to try to achieve an "oscillating blade" design. We tried a number of blade designs before finding one that would oscillate satisfactorily $(5,6)$. This design is based on a cantilever-mounted steel leaf spring with a plastic blade mounted at its end, as shown in Fig. 5. The main part of the blade is an inert plastic sheet of dumbbell shape. Square piezoelectric polymer sheets are mounted on each side of the plastic blade where it attaches to the leaf spring, which is the location of maximum bending of the plastic blade. Accordingly, as with the lateral blade design, the oscillating blade design has piezoelectric material in the outer fibers where the strain and thus the induced field are great, and inert material near the center fibers where the strain is small. This saves on expensive piezoelectric material without reducing performance appreciably.

The operation of this oscillating blade design can be visualized as two coupled oscillations $90^{\circ}$ out of phase. First, the cantilevered steel leaf spring oscillates upwind and downwind. Then, the composite plastic blade mounted at the leaf spring tip also oscillates upwind and downwind in a special manner which provides the net mechanical energy input needed for mechanical oscillation. When the leaf spring is moving downwind with maximum speed, the plastic blade is almost flat and presents maximum area to the wind, providing

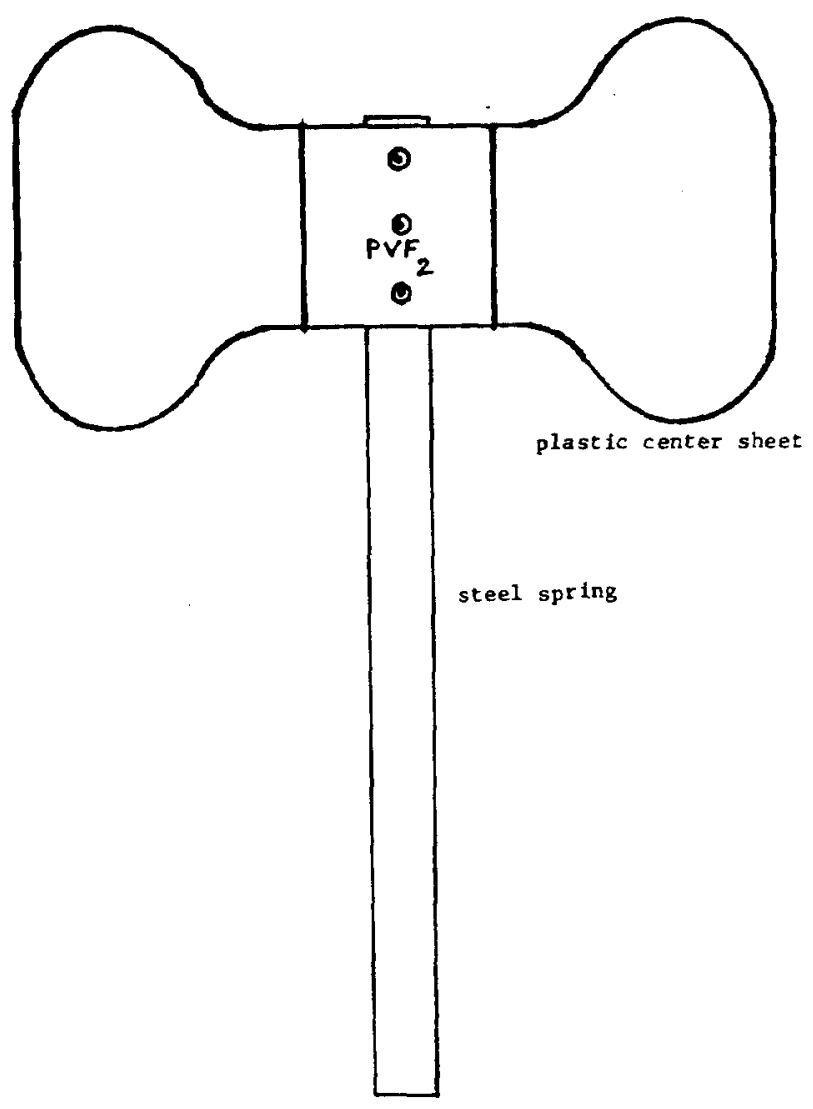

Fig. 5. Oscillating blade generator

maximum wind force even though the wind pressure is minimum because the blade is moving downwind. This force is in the direction of motion and so aids the oscillation buildup. When the leaf spring is moving upwind with maximum speed, the plastic blade has maximum curvature into a $U$ shape with the tips pointing away from the wind, so the projected area is small and the wind force is minimum but the pressure is maximum because of the upwind motion. This minimum force opposes the oscillation buildup, but it is smaller than the force on the other half of the cycle which aids buildup, so the oscillation builds up to an equilibrium amplitude.

We tested this design both in the wind tunnel and on a frame holding 16 generators and mounted on a flatbed truck, high enough to avoid the airstream from the cab. This design oscillated well for wind speeds of 10 to $25 \mathrm{~m} / \mathrm{s}$ (20 to 50 $\mathrm{mph}$ ), at its natural frequency of $30 \mathrm{~Hz}$ for our blade which is $12 \mathrm{~cm}$ tip-to-tip and has $9 \mathrm{~cm}^{2}$ area for each of the $\mathrm{PVF}_{2}$ squares composing the bimorph. The open-circuit output voltage 
exceeded 100 volts, but because the capacitive source impedance at $30 \mathrm{~Hz}$ was several megohms, the power output was only a few milliwatts. The peak strain was only $10 \%$ of yield strain, so the power would be 100 times greater if oscillation up to yield strain could be achieved. If electrical resonance could also be provided, the power would go up a factor of $Q$. A third way to increase power would be to cut size and thus boost resonant frequency; the power would be proportional to frequency if all other parameters remain the same. The practicality of achieving these improvements is the topic of the following Section.

\section{PROPOSED IMPROVEMENTS}

The above oscillating blade generator, in spite of eliminating the complexities of a rotating design, is still far too complicated and expensive for the power it can produce. To improve output per unit volume of $\mathrm{PVF}_{2}$ requires higher frequency operation, which requires smaller size, which is bad from the economy-of-scale standpoint. How can this dilemma be avoided?

To retain the oscillating instead of rotating feature and increase the oscillation frequency, it seems necessary to have a frame which could have many scaled-down oscillators of the general design described above, possible molded as a single piece of piezoelectrically inert plastic. There are other methods of inducing oscillations by air flow, described systematically in a book by Blevins (7). The piezoelectric material then would be in the support on which the frame is mounted. It could be in the form of bimorphs or multimorphs which bend, but for this type of design the direct compressional mode seems preferable. The oscillation frequency could be scaled up 30 times, to $1 \mathrm{kHz}$, by reducing the dimensions 30 times, from $12 \mathrm{~cm}$ to $4 \mathrm{~mm}$ tip-to-tip. Oscillation of a blade this size through $1 \mathrm{~mm}$ amplitude might impart a $0.1 \mathrm{~mm}$ amplitude vibration to the frame. At $1 \%$ operating strain in the compression mode, this would require $1 \mathrm{~cm}$ thickness of the piezoelectric polymer layer, a practical value if several sheets are stacked together. With a large amount of piezoelectric polymer having a large capacitance and operating at $1 \mathrm{kHz}$, it would be easy to resonate with a practical size inductor and greatly reduce the source impedance.
It should also prove practical to operate near the desired maximum strain and still avoid exceeding the yield strain in high winds. The safety problem of a rapidly turning rotor is eliminated, but there is the problem of a structure vibrating in the middle of the human audio range.

\section{CONCLUSIONS}

It may be feasible to design a wind generator based on piezoelectric polymers such as $\mathrm{PVF}_{2}$ which will operate at $1 \mathrm{kHz}$ in the compressional mode at a maximum strain of $1 \%$. Employing electrical resonance with a $Q$ of 30 , including losses both in the piezoelectric material and the inductor, it is theoretically possible to achieve power output of order 100 watts per $\mathrm{cm}^{3}$ of material. If a wind power plant because of the wind's intermittency should have a cost not exceeding \$1,000 per kilowatt capacity, and if the polymer constitutes $10 \%$ of the plant's cost, this means that the polymer should cost no more than $\$ 10 / \mathrm{cm}^{3}$. This is an attainable figure in mass production.

*Work supported by Montana Dept. of Natural Resources and Conservation, and DOE Grant DEFG06-87ER45292.

(1) J. Glanz, R\&D Magazine, Aug. 1992, p. 37.

(2) V.H. Schmidt, Proc. 6th IEEEInternat. Symp. on Appl. of Ferroelectrics, Bethlehem, PA, 1986, p. 538, IEEE Publ. CH2358-0/86/00000538.

(3) V.H. Schmidt, Proc. 1982 Wind and Solar Energy Technology Conference, Kansas City, MO.

(4) V.H. Schmidt, M. Klakken, and H. Darejeh, Proc. 6th Bien. Wind Energy Conf. and Workshop, Minneapolis, 1983, Am. Solar Energy Soc. Publ. 0737-3376/83, p. 315.

(5) H. Darejeh, M.S. Thesis, Montana State University, 1983.

(6) V.H. Schmidt, U.S. Patent No. 4,536,674, Aug. 20, 1985.

(7) R.D. Blevins, Flow-Induced Oscillations (Van Nostrand Reinhold Co., New York, 1977). 